\title{
Excluded-volume effects in linear polymers: Universality of generalized self-avoiding walks
}

\author{
R. Dekeyser \\ Instituut voor Theoretische Fysica, Katholieke Universiteit Leuven, B-3030, Belgium \\ A. Maritan \\ Dipartimento di Fisica della Università di Padova, 1-35100 Padova, Italy and \\ International School for Advanced Studies, Trieste, Italy and \\ Istituto Nazionale di Fisica Nucleare, Sezione di Padova, Italy \\ A. L. Stella \\ Dipartimento di Fisica della Università di Padova, I-35100 Padova, Italy and \\ International School for Advanced Studies, Trieste, Italy and \\ Istituto Nazionale di Fisica Nucleare, Sezione di Padova, Italy and \\ Unità Gruppo Nazionale di Struttura della Materia del Consiglio \\ Nazionale delle Ricerche, Padova, Italy \\ (Received 1 November 1984)
}

\begin{abstract}
A random walk which can visit each lattice site at most twice is considered. The universality of selfavoiding-walk critical behavior with respect to variations of a fugacity for self-intersections is predicted on the basis of general renormalization-group arguments and explicitly tested in two dimensions, both by exact enumeration analysis and by cluster scaling calculations. The meaning of the above universality and its consequences, as far as a correct formulation of Flory approximations is concerned, are briefly discussed.
\end{abstract}

The excluded-volume effect present in all real polymers in solution is expected to be crucial in determining the observed scaling conformational properties. Much theoretical effort has been devoted, both in proposing lattice randomwalk models able to simulate the above scaling properties, and in understanding up to which extent different ways of modeling an excluded-volume condition can lead to universal results for the critical behavior.

The simplest and most famous model giving a reasonable descritpion of the asymptotic properties of long polymers in a good solvent is the well known self-avoiding walk (SAW). ${ }^{1}$ Alternative descriptions with excluded volume effects are given by the model of Domb and Joyce ${ }^{2}$ and by the $v$-vertex trail. ${ }^{3}$ Investigations by series analysis ${ }^{2}$ and renormalization-group considerations ${ }^{4}$ suggest that these latter walks should belong to the SAW universality class.

More recently, particular attention has been given to the $k$-tolerant walk, ${ }^{3}$ a random walk in which each lattice site can be visited at most $k$ times (the SAW thus corresponding to $k=1$ ). A generalization of the Flory approximation ${ }^{1,5}$ has been proposed, ${ }^{6}$ which predicts a $k$-dependent scaling behavior for $k$-tolerant walks. On the other hand, series analysis $^{7,8}$ gave evidence for universality of the critical behavior for different $k$ values, for dimensionality $d=2$ and $d=3$.

In order to clarify the mechanism of the above universality and to understand better the failure of approximations of the Flory type, ${ }^{6}$ we concentrate here on the particular case of the 2-tolerant walk in $d=2$, generalized with an extra fugacity, in such a way to allow for a control of the average number of self-intersections.

In our model, we associate to each 2-tolerant walk of $n$ steps and $i$ (double) intersections a weight $K^{n} p^{i}$, in a grandcanonical formulation. For $p=0$ we recover the SAW and for $p=1$ we get the simple 2-tolerant walk. Self- intersections are favored for $p>1$, whereas they are disfavored for $p<1$. In this sense the 2-tolerant walk is a border case. Our generating function for the numbers $c_{n i}$ of $n$-step 2-tolerant walks with $i$ intersections, starting from a given origin, will be

$$
\chi(K, p)=\sum_{n, i} c_{n i} K^{n} p^{i} .
$$

The average radius of gyration is defined as usual by

$$
\xi^{2}(K, p)=\chi^{-1} \sum_{n, i} c_{n i} K^{n} p^{i} R_{n i}^{2},
$$

where $R_{n i}^{2}$ is the mean-square radius of gyration for $n$-step 2-tolerant walks with $i$ intersections.

The idea of the cluster renormalization group 9,10 is to rescale the lattice by a factor $l$, such that the new correlation length $\xi$, which for $K$ approaching some critical value $K_{c}(p)$ from below is expected to behave as $\xi \sim A\left[K_{c}(p)-K\right]^{-\nu}$, scales as

$$
\xi\left(K^{\prime}, p^{\prime}\right)=\frac{1}{l} \xi(K, p),
$$

where $K^{\prime}(K, p)$ and $p^{\prime}(K, p)$ are regular functions of $K$ and p.

We simply state the rules for obtaining the above mapping in our case (more details about the general approach can be found in the literature). ${ }^{9-12}$ Let us consider a partition of a square lattice in $l \times l$ cells. The renormalized fugacities $K^{\prime}$ and $p^{\prime}$ can be given by

$$
K^{\prime}=\sum_{W} K^{n(W)} p^{i(W)}
$$

and

$$
K^{\prime 2} p^{\prime}=\sum_{W, W^{\prime}} K^{n(W)} p^{i(W)} K^{n\left(W^{\prime}\right)} p^{i\left(W^{\prime}\right)} p^{I\left(W, W^{\prime}\right)},
$$


where the first sum is over all walks $W$ with $n(W)$ steps and $i(W)$ intersections, starting at a given point of the boundary of the cell (e.g., the lower left corner) and going across it in a given direction. The second sum runs over all pairs of walks of the same type as above, which cross the cell in perpendicular directions, without generating triple or quadruple intersections. The two walks $W$ and $W^{\prime}$ can have a number of mutual intersection points, $I\left(W, W^{\prime}\right)$ (including the starting point), as they are meant to belong to the same 2-tolerant walk; Eq. (5) should indeed be seen as the result of the coarse graining of a walk which returns to a given point after a relatively large number of steps. Explicit calculations show that slight modifications of these rules do not change the qualitative features of the phase diagram consistent with Eqs. (4) and (5).

From the above rules it is evident that the $p=0$ line (SAW) is an invariant subset under the mapping (4)-(5), with the pure SAW fixed point given by

$$
K^{*}=\sum_{W ; i(W)=0} K^{* n(W)}
$$

Furthermore, one easily realizes that $p^{\prime}<p$, at least for all $p \leqslant 1$. Indeed, due to the mutual starting point of $W$ and $W^{\prime}$ in Eq. (5), we know that $I\left(W, W^{\prime}\right) \geqslant 1$. Since each term in (5) corresponds to one of the terms contained in the square of (4), multiplied by $p^{I\left(W, W^{\prime}\right)}$, we may thus write for $p \leqslant 1: K^{\prime 2} p^{\prime} \leqslant K^{\prime 2} p$. This inequality becomes even more pronounced by the fact that we have to exclude from (5) those pairs of walks $W$ and $W^{\prime}$ that give rise to triple or higher intersection points inside the cell.

As explicit calculations show below, we thus have to expect that the $p=1$ line is not an invariant set of the transformation, and that the critical 2-tolerant walk is mapped under renormalization into a critical SAW problem.

Similar conclusions can be drawn for the $k$-tolerant walk with $k \geqslant 2$ and general $d$. Obviously, such conclusions are based on a renormalization scheme which is affected by intrinsic approximations. Further support for these conclusions comes from the comparison of the renormalization-group results with independent results obtained from series analysis, as presented bèlow.

For the $d=2, k=2$ case, we performed explicitly the cal-

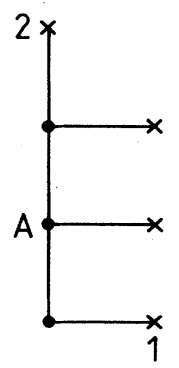

0

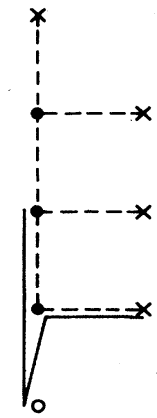

(b)

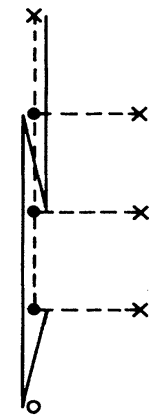

(c)

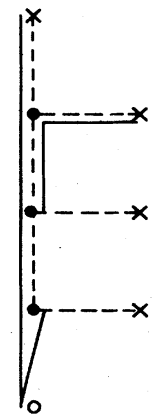

(d)
FIG. 1. (a) Anisotropic $3 \times 1$ cell, used for obtaining the recursion Eqs. (6)-(8). In (b), (c), and (d) we show some graphs contributing to the renormalized fugacities $K_{1}^{\prime}, K_{2}^{\prime}$, and $p^{\prime} K_{1}^{\prime} K_{2}^{\prime}$, respectively, using the rules suggested by Hoye and Napiórkowski (Ref. 13) for the one-dimensional random walk. culation of a transformation of the type (4)-(5). For simplicity we used an anisotropic cell rescaling with $l=3 .^{11,12}$ This corresponds to rescaling the lattice successively one direction at a time, using the $3 \times 1$ cell in Fig. 1 . The rules for renormalizing $K$ and $p$ are essentially the same ones as described above in the isotropic case; the recursion relations, however, are now different for the fugacity of bonds perpendicular $\left(K_{1}^{\prime}\right)$ and parallel $\left(K_{2}^{\prime}\right)$ to the rescaling direction. In the spirit of the Hoye and Napiórkowski calculations, ${ }^{13}$ in the recursion equations for $K_{1}^{\prime}$ and $K_{2}^{\prime}$ we count also the contribution of walks which go in the interior sites of the cell below the considered cell (open circles in Fig. 1). When the walk arrives at the boundary sites in the cell (crosses in Fig. 1), it stops. For renormalizing $K_{1}^{\prime}$ we choose, more symmetrically, the central point ( $A$ in Fig. 1) as the starting point for the transverse walks. Some examples of graphs contributing to $K_{1}^{\prime}, K_{2}^{\prime}$, and $p^{\prime} K_{1}^{\prime} K_{2}^{\prime}$ are also shown in Fig. 1.

The resulting recursion equations are

$K_{1}^{\prime}=K_{1}\left(1+2 K_{2}+2 K_{2}^{2} p+3 K_{2}^{3} p+2 K_{2}^{3} p^{2}+K_{2}^{4} p^{2}+2 K_{2}^{5} p^{2}\right)$,

$K_{2}^{\prime}=K_{2}^{3}\left(1+K_{2}^{2} p+2 K_{2}^{2} p^{2}+K_{2}^{4} p^{2}+K_{2}^{4} p^{3}+K_{2}^{6} p^{4}\right) ，$

$p^{\prime} K_{1}^{\prime} K_{2}^{\prime}=K_{1} K_{2}^{3} p\left[1+2 K_{2} p+K_{2}^{2} p\left(1+K_{2}^{2} p\right)\left(1+K_{2} p\right)\right]$.

In order to obtain an isotropic recursion, we first apply Eq. (6) and then Eq. (7) to the original fugacity $K$. This additional approximation is actually not very crude and the quantitative results are very little affected by changing the order of application of the two transformations. ${ }^{11,12}$ The renormalized $p$ is the value obtained after iterating Eqs. (6)-(8) twice, rescaling successively the $x$ and $y$ directions, and using $K_{1}=K_{2}=K$ as starting step fugacity.

In Fig. 2 we report the flow diagram resulting from our recursion scheme, while values of $K_{c}$ for different values of $p$ are reported in Table I, together with the $\nu$ exponent of the SAW fixed point, obtained from the relation

$$
\nu^{-1}=\left.\ln \frac{\partial K^{\prime}}{\partial K}\right|_{K^{*}, p=0} / \ln l \text {. }
$$

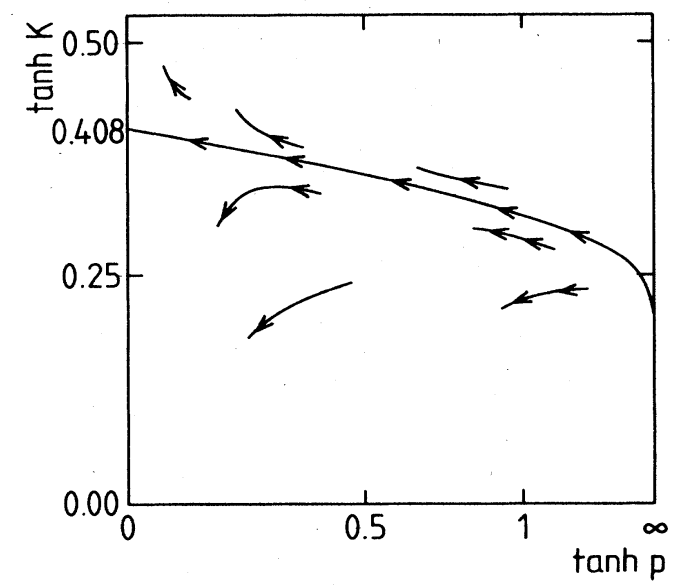

FIG. 2. Flow diagram obtained by our renormalization scheme for the grand-canonical formulation of the 2-tolerant walk for $d=2$ (square lattice). 
TABLE I. For different values of $p$ (first row), the values for $K_{c}$ and for the exponent $\nu$, as obtained from our renormalization scheme, are reported in the second and third row, respectively. In the last two rows, the values for $K_{c}$ and $2 \nu$ are reported, as obtained from the series analysis method.

\begin{tabular}{llllllll}
\hline \hline \multicolumn{1}{c}{$p$} & 0.0 & 0.1 & 0.5 & 0.9 & 1.0 & 1.1 & 1.5 \\
\hline$K_{c}$ (renormalization) & 0.408 & 0.396 & 0.349 & 0.311 & 0.303 & 0.296 & 0.272 \\
$\nu$ (renormalization) & 0.747 & 0.747 & 0.747 & 0.747 & 0.747 & 0.747 & 0.747 \\
$K_{c}$ (series) & 0.379 & 0.369 & 0.328 & 0.296 & 0.289 & 0.283 & 0.260 \\
$2 \nu$ (series) & 1.50 & 1.50 & 1.51 & 1.50 & 1.50 & 1.48 & 1.45 \\
& \pm 0.02 & \pm 0.02 & \pm 0.01 & \pm 0.01 & \pm 0.01 & \pm 0.02 & \pm 0.05 \\
\hline \hline
\end{tabular}

In order to confirm the results of our renormalizationgroup analysis, we performed exact enumerations (up to 16 steps on the square lattice) of the 2-tolerant walk for values of $p$ between 0 and 1.5. To reduce computing time, we have worked in Eq. (2) with the mean-square end-to-end distance, instead of the mean-square radius of gyration. The assumption is that

$$
\sum_{i} c_{n i} p^{i} \sim K_{c}^{-n} n^{\gamma-1}
$$

and

$$
\sum_{i} c_{n i} p^{i} R_{n i}^{2} \sim K_{c}^{-n} n^{\gamma_{R}-1} .
$$

This would imply that $\xi^{2}$ behaves as

$$
\xi^{2}(K, p) \sim\left(K_{c}-K\right)^{\gamma-\gamma_{R}},
$$

and thus $2 \nu=\gamma-\gamma_{R}$. The analysis of $\xi^{2}$ suffers from the numerical uncertainty on $K_{c}$, and this may be circumvented by analyzing instead the function

$$
\Lambda^{2}(K, p)=\sum_{n} K^{n}\left(\sum_{i} c_{n i} p^{i} R_{n i}^{2} / \sum_{i} c_{n i} p^{i}\right)
$$

for which we must expect

$$
\Lambda^{2}(K, p) \sim(1-K)^{\gamma-\gamma_{R}-1} .
$$

The singular behavior of $\Lambda^{2}, \xi^{2}, \chi$, and $\left(\chi \xi^{2}\right)$ as functions of $K$ has been analyzed by various standard methods ${ }^{14}$ (Néville tables, Padé analysis of logarithmic derivative,...) and by the recurrence relation method. ${ }^{15-17}$ All these methods give values of $\nu$ in good agreement with the SAW value $\nu=0.75,^{18}$ for all values of $p$ considered. In Table I we report, in particular, the values obtained by the last mentioned method of analysis, which revealed to be the most consistent. In the same table, we also report the estimates of $K_{c}(p)$, as obtained from analysis of $\chi$ and $\xi^{2}$. These values compare rather well with the renormalizationgroup values, and thus give further support to the validity of our conclusions. ${ }^{19}$

The model that we have introduced, which includes the 2-tolerant walk as a particular case, is thus in the universali- ty class of the SAW. ${ }^{20}$ In particular, this also holds for $p$ much larger than 1. Intersections are favored for large values of $p$, and at first sight it might seem contradictory to still find a SAW behavior. Consistently with the renormalization-group flow, however, we have to conclude that the tendency of the walk to have many intersections leads to the formation of many loops at a small scale, such that the walk becomes essentially a self-avoiding path at a coarse-grained level. When dealing with a $k$-tolerant walk in general, our guess is that multiple points in the statistically relevant configurations develop mostly at short distances along the chain, whereas a kind of self-avoiding constraint is guaranteed at longer distances (by the finiteness of $k$ ).

We may now ask what lessons can be drawn, from the above considerations, about the Flory approximation for $k$ tolerant walks. For a SAW of $n$ steps with radius of gyration $R$, this approximation consists ${ }^{1}$ in writing the free energy as

$$
F=A \frac{R^{2}}{n}+B \frac{n^{2}}{R^{d}},
$$

where the first term is the elastic part of the free energy and the second term is the repulsive energy, proportional to the number of monomers, multiplied with their concentration $n R^{-d}$. $A$ and $B$ are suitable dimensional factors. Minimization of (15) with respect to $R$ leads to the well known result $\nu=3 /(d+2)$ for $d \leqslant 4$. When $p \geqslant 1$, one might be tempted to suppose that the repulsive energy should be modified into something proportional to $\left(n R^{-d}\right)^{k+1} R^{d}$ for $k$-tolerant walks. $^{6}$ Such an expression would take into account the excluded-volume effects that start to be felt when $k+1$ monomers come close together. This would imply $\nu=(k+2) /(k d+2)$ for $d \leqslant d_{c}(k) \equiv 2(k+1) / k^{6}$ in contradiction with our result and, for $p=1$, with series results. $^{7,8}$ The actual excluded-volume mechanism determining the large scale behavior of the model is the one which prevents different, large segments of the walk (each one having many intersections at small scale) to come close together, such that, in a kind of coarse-grained sense, we have to conclude that the actual value of $k \geqslant 1$ is irrelevant. The appropriate form of the Flory free energy remains thus the same as given in Eq. (15).
1P. G. de Gennes, Scaling Concepts in Polymer Physics (Cornell Univ. Press, Ithaca, 1979).

${ }^{2}$ C. Domb and G. S. Joyce, J. Phys. C 5, 956 (1972).

${ }^{3}$ A. Malakis, J. Phys. A 9, 1283 (1976).

${ }^{4}$ F. Family, Phys. Lett. 92A, 341 (1982); F. Family and H. Gould, J.
Chem. Phys. 80, 3892 (1984); Y. Shapir and Y. Oono, J. Phys. A 17, L39 (1984).

${ }^{5}$ P. J. Flory, Principles of Polymer Chemistry (Cornell Univ. Press, Ithaca, 1969).

${ }^{6}$ L. Turban, J. Phys. A 16, L643 (1983). 
${ }^{7}$ A. J. Guttmann, C. Byrnes, and N. E. Frankel, J. Phys. A 17, L457 (1984).

${ }^{8}$ A. L. Stella (unpublished).

${ }^{9}$ H. E. Stanley, P. J. Reynolds, S. Redner, and F. Family, in Real Space Renormalization, edited by T. W. Burkhardt and J. M. J. van Leeuwen (Springer, Heidelberg, 1982).

${ }^{10} \mathrm{~F}$. Family, in Random Walks and their Applications in the Physical and Biological Sciences, edited by M. F. Shlesinger and B. J. West, AIP Conf. Proc. No. 109 (AIP, New York, 1984), pp. 33-72.

${ }^{11}$ A. Maritan and A. L. Stella, in Proceedings of VIII Sitges Conference on "Applications of Field Theory to Statistical Mechanics," edited by L. Garrido (Springer, Berlin, to be published).

${ }^{12}$ A. Maritan and A. L. Stella (unpublished).

13J. S. Hoye and M. Napiórkowski, J. Phys. A 13, 1897 (1980).

${ }^{14}$ D. S. Gaunt and A. J. Guttmann, in Phase Transitions and Critical Phenomena, edited by C. Domb and M. S. Green (Academic, New York, 1974), Vol. 3.

${ }^{15}$ A. J. Guttmann and G. S. Joyce, J. Phys. A 5, L81 (1972).

${ }^{16}$ A. J. Guttmann, J. Phys. A 81081 (1975).

${ }^{17}$ J. J. Rehr, G. S. Joyce, and A. J. Guttmann, J. Phys. A 13, 1587 (1980).

${ }^{18}$ B. Nienhuis, Phys. Rev. Lett. 49, 1062 (1982).
${ }^{19}$ For completeness, we should mention that direct analysis of $\chi$ and $\left(\chi \xi^{2}\right)$ seems to indicate that $\gamma$ and $\gamma_{R}$ are less independent of $p$ than their difference $2 \nu$. We find, e.g., $\gamma(p=0)=1.34$, $\gamma(p=1) \simeq 1.39, \gamma_{R}(p=0) \simeq 2.83, \gamma_{R}(p=1) \simeq 2.89$, with intermediate values for intermediate $p$. It is not clear whether this is due to numerical uncertainties or to a real deviation from universality for these quantities. It should be noted that the cluster renormalization-group argument deals only with $\xi$, and thus strictly speaking predicts universality only for the exponent $\nu$.

${ }^{20}$ Our conclusion is thus in sharp disagreement with the result obtained by F. Family [Phys. Lett. 92A, 341 (1982)]. On the basis of a simple renormalization argument, and unconfirmed by series expansions, this author claims to find universality only under the condition that no bond may be visited twice. In our model, the restriction is on the number of visits to the lattice sites. In a previous paper by J. A. Marqusee and J. M. Deutch [J. Chem. Phys. 75,5179 (1981)], it was argued that also these so-called $k$ tolerant trails, with restrictions on the bonds, do not exhibit universality. In view of intrinsic weaknesses of the arguments, we seriously doubt the correctness of their results, which are not confirmed by series analysis. 